\title{
Thermodynamic perturbation theory for polydisperse colloidal suspensions using orthogonal polynomial expansions
}

\author{
Sabine Leroch, ${ }^{1}$ Gerhard Kahl, ${ }^{1,2}$ and Fred Lado ${ }^{3}$ \\ ${ }^{1}$ Institut für Theoretische Physik, TU Wien, Wiedner Hauptstraße 8 - 10, A-1040 Wien, Austria \\ ${ }^{2} \mathrm{CMS}, \mathrm{TU}$ Wien, Wiedner Hauptstraße 8 - 10, A-1040 Wien, Austria \\ ${ }^{3}$ Department of Physics, North Carolina State University, Raleigh, North Carolina 27695
}

(Received 30 November 1998)

\begin{abstract}
We present a method for calculating the thermodynamic and structural properties of a polydisperse liquid by means of a thermodynamic perturbation theory: the optimized random phase approximation (ORPA). The approach is an extension of a method proposed recently by one of us for an integral equation application [Phys. Rev. E 54, 4411 (1996)]. The method is based on expansions of all $\sigma$-dependent functions in the orthogonal polynomials $p_{i}(\sigma)$ associated with the weight function $f_{\Sigma}(\sigma)$, where $\sigma$ is a random variable (in our case the size of the particles) with distribution $f_{\Sigma}(\sigma)$. As in the one-component or general $\mathcal{N}$-component case, one can show that the solution of the ORPA is equivalent to the minimization of a suitably chosen functional with respect to variations of the direct correlation functions. To illustrate the method, we study a polydisperse system of square-well particles; extension to other hard-core or soft-core systems is straightforward. [S1063-651X(99)06806-3]
\end{abstract}

PACS number(s): 82.70.Dd, 02.70.-c, 61.20.Gy, 61.25.Em

\section{INTRODUCTION}

Colloidal suspensions consist of mesoscopic particles (with mean diameters typically from $1 \mu \mathrm{m}$ to $1 \mathrm{~nm}$ ) that are dispersed in a suspending microscopic fluid. On the one hand, the fact that such colloids are practically ubiquitous in our everyday lives (ranging from industrial products such as paints, glues, or lubricants to basics such as foods or pharmaceuticals) is one reason why increasing attention has been dedicated to these systems. (For an overview see, for instance, Ref. [1].) On the other hand, for a liquid state physicist such systems are very attractive in that they represent mesoscopic realizations of simple atomic liquids. Surprising analogies between the statistical behavior of such systems and that of simple atomic fluids can be observed, and in some cases analogous experiments can be carried out much more easily for colloids than for atomic systems. To describe these suspensions, one usually integrates out (at least conceptually) the molecular degrees of freedom of the suspending fluid, and employs effective potentials acting directly between the mesoscopic particles that are rather simple: a harsh repulsion at short distances followed by attraction at larger distances. In some cases, these interactions can even be tailored by suitable production techniques, while in atomic liquids one is simply stuck with the interaction dictated by the electronic structure.

These appealing features of colloidal suspensions are countered by (at least) one major drawback which makes their theoretical investigation rather complicated: due to their production process the colloidal particles are inherently polydisperse in size (and consequently also in their interaction). A polydisperse liquid can be considered as a mixture with an infinite number of components, characterized, for instance, by size $\sigma$, which is now a continuous rather than a discrete variable. The first attempts to treat such systems date back to the late 1970s [2,3], when a model of polydisperse hard spheres was investigated within the Percus-Yevick approximation. In later years the concept of polydispersity was cast in a mathematically rigorous form by Salacuse and Stell [4] and by Briano and Glandt [5]. In these formulations, $\sigma$ is the realization of some random variable $\Sigma$ distributed according to a probability distribution $f_{\Sigma}(\sigma)$, which replaces the finite set of concentrations of the discrete components of a mixture. Meanwhile, the formalism has been extended systematically to more refined and more sophisticated hard-core model systems [6-9]. The first step toward numerically solving such general (i.e., continuous) systems was carried out by D'Aguanno and Klein [10], who applied well-known integral equation approaches of liquid state theory to the polydisperse case. This was achieved by replacing the continuous distribution $f_{\Sigma}(\sigma)$ with a histogram for a finite set of $n$ wellchosen diameters, thus mapping the polydisperse system back onto an $n$-component mixture.

Recently this approach was modified by one of us by joining the orthogonal polynomial expansion technique with classical liquid state integral equation theory [11]. In this procedure, all $\sigma$-dependent functions (in particular, the correlation functions) are expanded in terms of orthogonal polynomials $p_{i}(\sigma)$ associated with the weight function $f_{\Sigma}(\sigma)$. This expansion technique avoids the rapid increase in computational cost with number of components $n$ in the D'Aguanno-Klein mixtures method, while fully retaining the advantages and numerical accuracy of Gaussian quadrature based on the weight function $f_{\Sigma}(\sigma)$. The same technique can further be applied to a much wider range of problems dealing with internal and external degrees of freedom in fluid systems $[12,13]$.

In the present work, we have merged the orthogonal polynomial expansion method with a thermodynamic perturbation theory, the optimized random phase approximation 
(ORPA). The ORPA was introduced in the 1970s [14,15], and turned out to be a very successful liquid state theory, favored by practitioners over a considerable period of time until-because of new, more efficient numerical algorithms - it was overtaken by integral equations in the 1980s. In the ORPA, the pair potential is split into a harshly repulsive reference term (the optimum choice is of course a hard sphere interaction) and a perturbation term; the Ornstein-Zernike equations are then solved along with a mean-spherical-type closure relation which guarantees in addition that the pair distribution function vanishes in the region that is not accessible due to the strong repulsion ("core region"). It can be shown that the solution of the resulting integral equation is equivalent to the minimization problem of a suitably chosen functional of the direct correlation function. This functional makes the ORPA very attractive, since it is sometimes more convenient (and numerically more stable) to solve the minimization problem than to solve an integral equation. Thermodynamic and structural properties are calculated via perturbation expressions. In recent yearsdue in particular to the work of Pastore and co-workers $[16,17]$ - the ORPA has been rediscovered: new numerical tools and the rapid development of computers have brought this liquid state method back into the race. Preliminary calculations for simple square-well systems have shown that in some cases the ORPA can in fact be applied over a larger range of system parameters than integral equation approaches [18]. In addition, the ORPA shows a remarkable degree of thermodynamic consistency without the necessity of introducing an additional parameter, as required in applications of parametrized integral equation theories that enforce some thermodynamic consistency.

We demonstrate here that one can merge the ORPA and the method of orthogonal polynomial expansions. As a sample application, we have chosen a polydisperse system of square-well molecules. This is a rather simple system which nevertheless captures all the essential features of a typical potential of atomic liquids or colloidal suspensions. In this formulation, the potential parameters of the polydisperse system (hard-core diameter $\sigma$, well width $\lambda$, and well depth $\epsilon$ ) can be varied independently; i.e., they can be distributed according to three independent distribution functions $f_{\Sigma}(\sigma)$, $f_{\Lambda}(\sigma)$, and $f_{E}(\sigma)$.

Finally we note some very recent work on polydisperse system where contributions of higher-order moments of the distribution function to the free energy are systematically taken into account $[19,20]$. At present it is not clear if some relation between this moment-expansion and our expansion in orthogonal polynomials can be established; this might be part of future work.

The paper is organized as follows. In Sec. II, we present the basic concept of the ORPA for the case of a discrete $\mathcal{N}$-component system (with finite $\mathcal{N}$ ) as well as for the case of a continuous polydisperse system, introducing orthogonal polynomial expansions for the latter. General expressions for the structural and thermodynamic properties are given here. In Sec. III, we apply this approach to a polydisperse squarewell liquid, and discuss the influence of the numerical parameters and of the size distribution functions. The paper concludes with a summary of our findings in Sec. IV.

\section{THEORY}

\section{A. System}

We first consider an $\mathcal{N}$-component mixture of simple liquids that is characterized by a temperature $T\left[\beta=\left(k_{B} T\right)^{-1}\right]$, a number density $\rho$, and a set of concentrations $c_{i}$, and define the partial number densities $\rho_{i}=\rho c_{i}$. We furthermore assume that every species $i$ is characterized by some diameter $\sigma_{i}$, and that the pair potential $\Phi\left(r ; \sigma_{i}, \sigma_{j}\right)$ acting between particles of species $i$ and $j$ can be split up into a repulsive reference part $\Phi_{0}\left(r ; \sigma_{i}, \sigma_{j}\right)$ and a perturbation part $\Phi_{1}\left(r ; \sigma_{i}, \sigma_{j}\right)$ :

$$
\Phi\left(r ; \sigma_{i}, \sigma_{j}\right)=\Phi_{0}\left(r ; \sigma_{i}, \sigma_{j}\right)+\Phi_{1}\left(r ; \sigma_{i}, \sigma_{j}\right) .
$$

We can assume that the reference potentials are hard sphere potentials, characterized by an additive set of diameters $\sigma_{i}$, and put

$$
\sigma_{i j}=\frac{1}{2}\left(\sigma_{i}+\sigma_{j}\right) .
$$

If the $\Phi_{0}\left(r ; \sigma_{i}, \sigma_{j}\right)$ are soft potentials, we can replace them by suitably chosen hard sphere potentials using a straightforward generalization of the Barker-Henderson [21] or the Weeks-Chandler-Andersen [15,22] prescription for the determination of the diameters; of course, in such a case the cross terms in the interactions must be forced to be additive. We will characterize our systems via the packing fraction $\eta$, defined as

$$
\eta=\frac{\pi}{6} \sum_{i=1}^{\mathcal{N}} \rho_{i} \sigma_{i}^{3}
$$

In our approach, we exploit the fact that the thermodynamic and structural properties of the hard sphere reference potential are well known, either as the (thermodynamically inconsistent) analytic Percus-Yevick solution [23] or as semiempirical Verlet-Weis-type extensions [24-26] which very accurately parametrize computer experiments.

\section{B. Optimized random phase approximation for the $\mathcal{N}$-component case}

We start from the Ornstein-Zernike (OZ) equation

$$
\begin{aligned}
h\left(r ; \sigma_{i}, \sigma_{j}\right)= & c\left(r ; \sigma_{i}, \sigma_{j}\right)+\sum_{k=1}^{\mathcal{N}} \rho_{k} \int d \mathbf{r}^{\prime} c\left(r^{\prime} ; \sigma_{i}, \sigma_{k}\right) \\
& \times h\left(\left|\mathbf{r}-\mathbf{r}^{\prime}\right| ; \sigma_{k}, \sigma_{j}\right),
\end{aligned}
$$

where $c\left(r ; \sigma_{i}, \sigma_{j}\right)$ and $h\left(r ; \sigma_{i}, \sigma_{j}\right)$ are the direct and total correlation functions, respectively. We follow Blum and Stell [3] and introduce

$$
\begin{aligned}
& H\left(r ; \sigma_{i}, \sigma_{j}\right)=\sqrt{\rho_{i} \rho_{j}} h\left(r ; \sigma_{i}, \sigma_{j}\right), \\
& C\left(r ; \sigma_{i}, \sigma_{j}\right)=\sqrt{\rho_{i} \rho_{j}} c\left(r ; \sigma_{i}, \sigma_{j}\right) .
\end{aligned}
$$

Fourier transforms are denoted by a tilde; introducing matrix notation, $[\mathbf{C}(r)]_{i j}=C\left(r ; \sigma_{i}, \sigma_{j}\right)$, etc., we arrive at the following $\mathrm{OZ}$ matrix equation in $q$ space:

$$
\widetilde{\mathbf{H}}(q)=\widetilde{\mathbf{C}}(q)+\widetilde{\mathbf{C}}(q) \widetilde{\mathbf{H}}(q) .
$$


Just as for the potentials, we now split the correlation functions $\mathbf{H}(r)$ and $\mathbf{C}(r)$ into reference and perturbation parts:

$$
\mathbf{C}(r)=\mathbf{C}_{0}(r)+\mathbf{C}_{1}(r), \quad \mathbf{H}(r)=\mathbf{H}_{0}(r)+\mathbf{H}_{1}(r) .
$$

Since $\{\mathbf{C}(r), \mathbf{H}(r)\}$ and $\left\{\mathbf{C}_{0}(r), \mathbf{H}_{0}(r)\right\}$ satisfy OZ relations, we arrive-after some matrix algebra-at the so-called residual $\mathrm{OZ}$ relation

$$
\widetilde{\mathbf{H}}_{1}(q)=\left\{\left[\mathbf{S}_{0}(q)\right]^{-1}-\widetilde{\mathbf{C}}_{1}(q)\right\}^{-1} \widetilde{\mathbf{C}}_{1}(q) \mathbf{S}_{0}(q),
$$

where we have introduced the matrix of structure factors of the reference system,

$$
\mathbf{S}_{0}(q)=\mathbf{1}+\widetilde{\mathbf{H}}_{0}(q),
$$

with 1 the unit matrix.

To solve the $\mathrm{OZ}$ equations a closure relation is needed. In the random phase approximation (RPA), one assumes that the long-range behavior of the direct correlation functions (DCF's) holds for all distances outside the core region,

$$
c\left(r ; \sigma_{i}, \sigma_{j}\right)=c_{0}\left(r ; \sigma_{i}, \sigma_{j}\right)-\beta \Phi_{1}\left(r ; \sigma_{i}, \sigma_{j}\right),
$$

or equivalently,

$$
c_{1}\left(r ; \sigma_{i}, \sigma_{j}\right)=-\beta \Phi_{1}\left(r ; \sigma_{i}, \sigma_{j}\right), \quad r>\sigma_{i j} .
$$

Due to the infinite repulsion of the reference potential, there is some ambiguity for the definition of $\Phi_{1}\left(r ; \sigma_{i}, \sigma_{j}\right)$ inside the core. One possibility is to assume that the perturbation potential is continued steadily. Solution of the RPA closure along with the $\mathrm{OZ}$ relation then leads, however, to pair distribution functions [PDF's, $g\left(r ; \sigma_{i}, \sigma_{j}\right)=h\left(r ; \sigma_{i}, \sigma_{j}\right)+1$ ] that violate the core conditions, i.e.,

$$
g\left(r ; \sigma_{i}, \sigma_{j}\right) \neq 0 \text { for } r<\sigma_{i j} .
$$

The optimized version of the RPA, ORPA, corrects this deficiency by modifying $c_{1}\left(r ; \sigma_{i}, \sigma_{j}\right)$ inside the core, where the perturbation potential (and hence the perturbation part of the DCF's) can be changed arbitrarily. The modifications are then done in such a way as to guarantee that the core condition for the PDF's is fulfilled. Hence the ORPA closure relations now read

$$
\begin{gathered}
c\left(r ; \sigma_{i}, \sigma_{j}\right)=-\beta \Phi_{1}\left(r ; \sigma_{i}, \sigma_{j}\right), \quad r>\sigma_{i j} \\
g\left(r ; \sigma_{i}, \sigma_{j}\right)=0, \quad r<\sigma_{i j} .
\end{gathered}
$$

Similarly to the one-component case, one can show that the solution of the integral equation along with the ORPA closure is equivalent to minimization of a functional $\mathcal{F}[\mathbf{C}]$ of the direct correlation functions. Defining

$$
\begin{aligned}
\mathcal{F}[\mathbf{C}]= & \frac{1}{2(2 \pi)^{3}} \int d \mathbf{q}\left\{\operatorname{Tr}\left[\widetilde{\mathbf{C}}_{1}(q) \mathbf{S}_{0}(q)\right]\right. \\
& \left.+\ln \left[\operatorname{det}\left(\mathbf{1}-\widetilde{\mathbf{C}}_{1}(q) \mathbf{S}_{0}(q)\right)\right]\right\},
\end{aligned}
$$

where $\operatorname{Tr}$ denotes the trace of a matrix, one can show that

$$
\frac{\delta \mathcal{F}[\mathbf{C}]}{\delta C_{1}\left(r ; \sigma_{1}, \sigma_{j}\right)}=-\frac{1}{2} H_{1}\left(r ; \sigma_{i}, \sigma_{j}\right) \text { for } r<\sigma_{i j} .
$$

This means that the search for the minimum of the functional $\mathcal{F}[\mathbf{C}]$ with respect to variations of the $C_{1}\left(r ; \sigma_{i}, \sigma_{j}\right)$ inside the core region is equivalent to satisfying the core condition for the PDF's. By extending a proof by Pastore et al. [16] to the $\mathcal{N}$-component case, one may show that, if such a minimum has been found, it is the only one and the physically sound one.

\section{Optimized random-phase approximation for the polydisperse case}

The formalism developed above can now be easily generalized to the polydisperse case. The concept of polydisperse systems was introduced in the late 1970s for simple hard spheres [3,27], and extended in subsequent years to more complex interaction models (see the Introduction of Ref. [11]). Later, the treatment of polydispersity was cast in a rigorous form by Salacuse and Stell [4] and Briano and Glandt [5]. In a polydisperse system (which may be viewed as a mixture with an infinite number of components), the particles are characterized by a parameter $\sigma$ which is the realization of a random variable $\Sigma$. This random variable is distributed according to some probability distribution $f_{\Sigma}(\sigma)$, which now replaces the set of concentrations of the components. In our case (as in many other applications), the random variable is of course the diameter of the particles. In the following we will set $f_{\Sigma}(\sigma)=f(\sigma)$ for convenience and reintroduce the index " $\Sigma$ " later.

The $\mathrm{OZ}$ equation now reads

$$
\begin{aligned}
h\left(r_{12} ; \sigma_{1}, \sigma_{2}\right)= & c\left(r_{12} ; \sigma_{1}, \sigma_{2}\right)+\rho \int_{0}^{\infty} d \sigma_{3} f\left(\sigma_{3}\right) \\
& \times \int d \mathbf{r}_{3} c\left(r_{13} ; \sigma_{1}, \sigma_{3}\right) h\left(r_{32} ; \sigma_{3}, \sigma_{2}\right),
\end{aligned}
$$

and the packing fraction $\eta$ is given by the third moment of the distribution $f(\sigma)$,

$$
\eta=\frac{\pi}{6} \rho \int_{0}^{\infty} d \sigma f(\sigma) \sigma^{3}=\frac{\pi}{6} \rho \overline{\sigma^{3}} .
$$

Note that in some papers a slightly different definition of the packing fraction is used, e.g. [4],

$$
\bar{\eta}=\frac{\pi}{6} \rho \bar{\sigma}^{3}, \quad \bar{\sigma}=\int_{0}^{\infty} d \sigma f(\sigma) \sigma .
$$

Instead of evaluating integrals over the distribution function $f(\sigma)$ by Gaussian quadrature, as proposed by D'Aguanno and Klein [10], we expand all $\sigma$-dependent functions in terms of the orthonormal polynomials $p_{i}(\sigma), i$ $=0,1,2 \ldots$, associated with the distribution function $f(\sigma)$, which now plays the role of a weight function,

$$
\int_{0}^{\infty} d \sigma f(\sigma) p_{i}(\sigma) p_{j}(\sigma)=\delta_{i j} .
$$

For some choices of $f(\sigma)$, such as the Schulz (or $\Gamma$ ) distribution used below, $p_{i}(\sigma)$ are known explicitly. In other 
cases, these polynomials must be constructed; this can be done using the Gram-Schmidt algorithm, starting from $p_{0}(\sigma)=1$.

Given now some $\sigma$-dependent functions $x(r ; \sigma)$ and $y\left(r ; \sigma_{1}, \sigma_{2}\right)$, their expansions read

$$
\begin{gathered}
x(r ; \sigma)=\sum_{j=0}^{\infty} x_{j}(r) p_{j}(\sigma), \\
y\left(r ; \sigma_{1}, \sigma_{2}\right)=\sum_{i, j=0}^{\infty} y_{i j}(r) p_{i}\left(\sigma_{1}\right) p_{j}\left(\sigma_{2}\right),
\end{gathered}
$$

where the coefficients are given by

$$
\begin{gathered}
x_{i}(r)=\int_{0}^{\infty} d \sigma f(\sigma) x(r ; \sigma) p_{i}(\sigma), \\
y_{i j}(r)=\int_{0}^{\infty} d \sigma_{1} d \sigma_{2} f\left(\sigma_{1}\right) f\left(\sigma_{2}\right) y\left(r ; \sigma_{1}, \sigma_{2}\right) p_{i}\left(\sigma_{1}\right) p_{j}\left(\sigma_{2}\right) .
\end{gathered}
$$

Of course, in numerical applications these infinite sums and integrations must be converted to finite versions. For the integrations, it is most convenient to use Gaussian quadrature. Let $\sigma_{k ; n}(k=1, \ldots, n)$ be the $n$ roots of $p_{n}(\sigma)$, and $z(\sigma)$ some arbitrary $\sigma$-dependent function; then

$$
\int_{0}^{\infty} d \sigma f(\sigma) z(\sigma) \approx \sum_{k=1}^{n} w_{k} z\left(\sigma_{k ; n}\right),
$$

where the weights $w_{k}$ are given by

$$
w_{k}=\frac{1}{\sum_{j=0}^{n-1} p_{j}^{2}\left(\sigma_{k ; n}\right)} .
$$

With these ingredients, we obtain the numerical (approximate) versions of the equations above:

$$
\begin{gathered}
x\left(r ; \sigma_{k ; n}\right) \approx \sum_{j=0}^{n-1} x_{j}(r) p_{j}\left(\sigma_{k ; n}\right), \\
y\left(r ; \sigma_{k ; n}, \sigma_{m ; n}\right) \approx \sum_{i, j=0}^{n-1} y_{i j}(r) p_{i}\left(\sigma_{k ; n}\right) p_{j}\left(\sigma_{m ; n}\right),
\end{gathered}
$$

where the coefficients are given by

$$
\begin{gathered}
x_{i}(r) \approx \sum_{k=1}^{n} w_{k} x\left(r ; \sigma_{k ; n}\right) p_{i}\left(\sigma_{k ; n}\right), \\
y_{i j}(r) \approx \sum_{k, m=1}^{n} w_{k} w_{m} y\left(r ; \sigma_{k ; n}, \sigma_{m ; n}\right) p_{i}\left(\sigma_{k ; n}\right) p_{j}\left(\sigma_{m ; n}\right) .
\end{gathered}
$$

Denoting the expansion coefficients for the orthogonal polynomial expansions of the direct and total correlation functions $c\left(r ; \sigma_{i}, \sigma_{j}\right)$ and $h\left(r ; \sigma_{i}, \sigma_{j}\right)$ by $c_{i j}(r)$ and $h_{i j}(r)$ (and similarly for their Fourier transforms, with an additional tilde) and collecting them as matrices $\mathrm{C}$ and $\mathrm{H}$, we find that in terms of these coefficient functions the $\mathrm{OZ}$ equations (6) now read

$$
\tilde{\mathrm{H}}(q)=\tilde{\mathrm{C}}(q)+\rho \tilde{\mathrm{H}}(q) \tilde{\mathrm{C}}(q) .
$$

Here, $\mathrm{C}$ and $\mathrm{H}$ are $n \times n$ matrices, where $n$ is the (chosen) parameter of discretization; the actual values for $n$ required for a sufficient level of accuracy are discussed below. In a similar way, we can decompose the static structure factors $S\left(q ; \sigma_{i}, \sigma_{j}\right)$ of the reference system in terms of the orthonormal polynomials, leading to a matrix $\mathrm{S}_{0}(q)$ that is related to the hard sphere PDF matrix $\tilde{\mathrm{H}}_{0}(q)$ via

$$
\mathrm{S}_{0}(q)=1+\rho \tilde{\mathrm{H}}_{0}(q) \text {. }
$$

Developing the concept of the ORPA along lines parallel to those outlined above for the $\mathcal{N}$-component case, we find that the residual OZ equation (8) now reads

$$
\tilde{\mathrm{H}}_{1}(q)=\left\{\left[\mathrm{S}_{0}(q)\right]^{-1}-\rho \tilde{\mathrm{C}}_{1}(q)\right\}^{-1} \tilde{\mathrm{C}}_{1}(q) \mathrm{S}_{0}(q) \text {. }
$$

Again, it can be shown that the solution of the residual OZ relation along with the ORPA closure are equivalent to minimizing a suitably chosen functional. In terms of the $\mathrm{C}$ and $\mathrm{S}_{0}$ matrices, the functional and its functional derivative with respect to the coefficients $\mathrm{C}_{1 ; i j}(r)$ are, respectively,

$$
\begin{aligned}
\mathcal{F}[\mathrm{C}]= & \frac{1}{2(2 \pi)^{3}} \int d \mathbf{q}\left\{\operatorname{Tr}\left[\rho \tilde{\mathrm{C}}_{1}(q) \mathrm{S}_{0}(q)\right]\right. \\
& \left.+\ln \left[\operatorname{det}\left(1-\rho \tilde{\mathrm{C}}_{1}(q) \mathrm{S}_{0}(q)\right)\right]\right\}
\end{aligned}
$$

and

$$
\frac{\delta \mathcal{F}[\mathrm{C}]}{\delta c_{1 ; i j}(r)}=-\frac{1}{2} \rho^{2} h_{1 ; i j}(r) \text { for } r<\sigma_{i j}
$$

\section{Thermodynamics}

Once the structure is found, the thermodynamic properties can easily be calculated. In the following, the expressions presented in Ref. [11] are complemented by some additional relations which can be derived specifically in the framework of the ORPA.

For the (excess) internal energy $U^{\mathrm{ex}}$, the virial pressure $P^{v}$, and the isothermal compressibility $\chi_{T}$, we thus obtain

$$
\begin{gathered}
\frac{\beta U^{\mathrm{ex}}}{N}=2 \pi \beta \rho \sum_{l, m} \int_{0}^{\infty} d r r^{2} \Phi_{1 ; l m}(r) g_{l m}(r), \\
\frac{\beta P^{v}}{\rho}=1-\frac{2}{3} \pi \beta \rho \sum_{l, m} \int_{0}^{\infty} d r r^{3} \frac{d \Phi_{l m}(r)}{d r} g_{l m}(r), \\
\frac{1}{\rho k_{B} T \chi_{T}}=\left(\frac{\partial \beta P^{c}}{\partial \rho}\right)_{T}=1-\rho \tilde{c}_{00}(q=0)
\end{gathered}
$$

where $N$ is the total number of particles of all species. Further, within the ORPA and using the coupling constant formalism [11], for the Helmholtz free energy $A$ one can derive the expression 
TABLE I. Parameters that define systems I and II investigated in this study: $\eta$ is the packing fraction as defined in Eq. (17); $\lambda$ is the $\sigma$-independent well width; $\alpha$ is the width parameter of the Schulz distribution [cf. Eq. (38); $\bar{\sigma}$ is set to unity]; $\bar{\epsilon}$ and $z$ are as defined in Eq. (42).

\begin{tabular}{lccccc}
\hline \hline & $\eta$ & $\lambda$ & $\alpha$ & $\bar{\epsilon}$ & $z$ \\
\hline System I & 0.30 & 1.25 & 100 & 1.0 & 1.5 \\
System II & 0.35 & 1.50 & 50 & 0.8 & 1.0 \\
\hline \hline
\end{tabular}

$$
\beta A=\beta A_{0}+\beta A^{\mathrm{HTA}}+\beta A^{\mathrm{ORPA}},
$$

where $A_{0}$ is the free energy of the polydisperse hard sphere reference system [3], and the other two contributions are

$$
\begin{gathered}
\beta A^{\mathrm{HTA}}=2 \pi N \rho \sum_{l, m} \int_{0}^{\infty} d r r^{2} \Phi_{1 ; l m}(r) g_{0 ; l m}(r), \\
\beta A^{\mathrm{ORPA}}=-\frac{N}{\rho} \mathcal{F}[\mathrm{C}] .
\end{gathered}
$$

In Sec. III, we will present results for $A^{\mathrm{ex}}=A-A_{\mathrm{id}}$, where $A_{\text {id }}$ is the ideal gas contribution.

\section{RESULTS}

\section{A. Schulz (or $\Gamma)$ distribution}

We assume that the particle diameter $\sigma$ is distributed according to a $\Gamma$ distribution [28],

$$
f_{\Sigma}(\sigma)=\left(\frac{\alpha+1}{\bar{\sigma}}\right)^{\alpha+1} \frac{\sigma^{\alpha} e^{-(\alpha+1) \sigma / \bar{\sigma}}}{\Gamma(\alpha+1)},
$$

where $\bar{\sigma}$ is the mean diameter and $\alpha$ is a positive parameter. The $\Gamma$ distribution is often used to describe polydisperse fluids; for us it has the further benefit that the orthonormal polynomials $p_{i}(\sigma)$ defined in Eq. (19) for the weight function $f_{\Sigma}(\sigma)$ are known explicitly-they are proportional to the associated Laguerre polynomials $L_{i}^{(\alpha)}(x)$,

$$
p_{i}(\sigma)=\left[\frac{i ! \Gamma(\alpha+1)}{\Gamma(i+\alpha+1)}\right]^{1 / 2} L_{i}^{(\alpha)}\left((\alpha+1) \frac{\sigma}{\bar{\sigma}}\right)
$$

For this distribution the expressions for the packing fractions introduced above now read

$$
\eta=\frac{\pi}{6} \rho \bar{\sigma}^{3} \frac{(\alpha+2)(\alpha+3)}{(\alpha+1)^{2}}, \quad \bar{\eta}=\frac{\pi}{6} \rho \bar{\sigma}^{3} .
$$

\section{B. System}

To demonstrate our method, we have chosen a polydisperse square-well liquid, where the interactions are given by

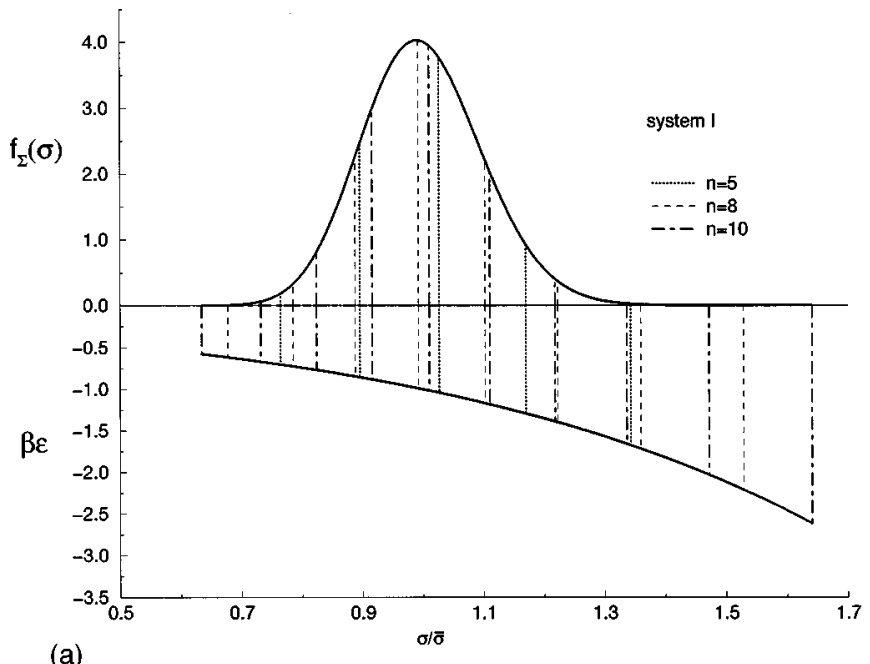

(a)

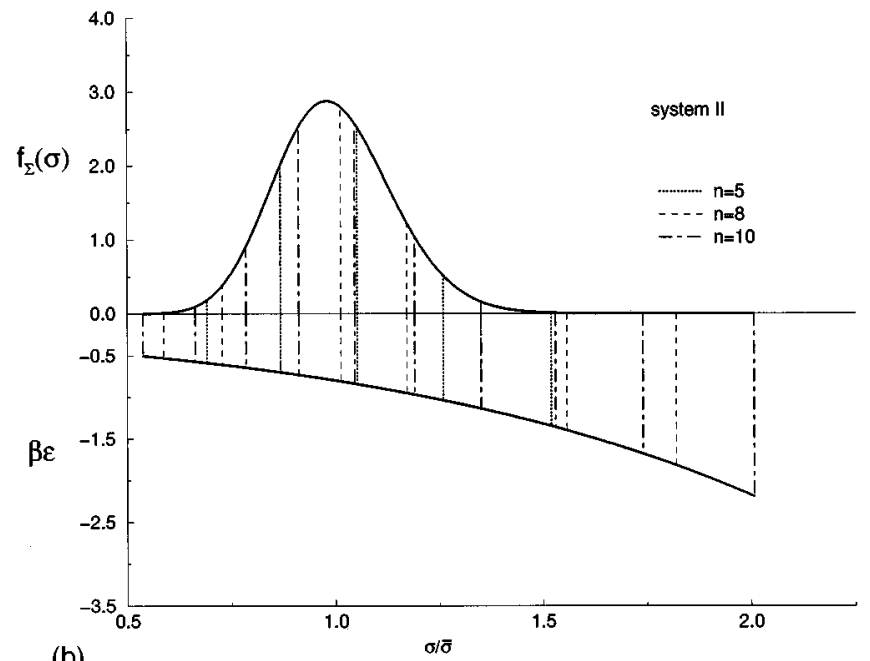

(b)

FIG. 1. Distribution function $f_{\Sigma}(\sigma)$ (in units $1 / \bar{\sigma}$ ) for the particle size of system I (a) and system II (b) for the two different polydisperse square-well systems considered in this study. The vertical lines locate the zeros $\sigma_{k ; n}$ of the orthogonal polynomials $p_{n}(\sigma)$ associated with the weight function $f_{\Sigma}(\sigma)$ for different values of $n$ ( $n=5$ : broken line; $n=8$ : dotted line; $n=10$ : full line). On the negative ordinate, we plot $-\beta \epsilon_{i i}=-\beta \epsilon\left(\sigma_{i}, \sigma_{i}\right)$, the parameters of the well depth of the "diagonal" interatomic potential, as a function of $\sigma$. For the unlike case, $\epsilon_{i j}$ and $i \neq j$, we have assumed a Berthelot rule, Eq. (42).

$$
\beta \Phi\left(r ; \sigma_{i}, \sigma_{j}\right)= \begin{cases}\infty, & r \leqslant \sigma_{i j} \\ -\epsilon_{i j}, & \sigma_{i j}<r \leqslant \lambda_{i j} \sigma_{i j} \\ 0, & \lambda_{i j} \sigma_{i j}<r .\end{cases}
$$

Here $\epsilon_{i j}$ are the parameters for the well depth, and $\lambda_{i j}$ the parameters for the range of the well. These quantities allow us to take into account-independently from each otherpolydispersity in particle size, interaction strength, and potential range.

In particular, we demonstrate our method for two systems, denoted I and II. Their system parameters are compiled in Table I, and have the following meaning: $\eta$ is the packing fraction as defined in Eq. (17), while the parameters $\bar{\epsilon}$ and $z$ define the $\sigma$ dependence of the well depth, 


$$
\epsilon_{i i}=\epsilon\left(\sigma_{i}, \sigma_{i}\right)=\bar{\epsilon} e^{z\left(\sigma_{i}-\bar{\sigma}\right)}, \quad \epsilon_{i j}=\epsilon\left(\sigma_{i}, \sigma_{j}\right)=\bar{\epsilon} e^{z\left(\sigma_{i j}-\bar{\sigma}\right)},
$$

$\bar{\sigma}$ being the mean diameter, i.e., the first moment of $f_{\Sigma}(\sigma)$. This simple choice for the $\sigma$ dependence of the well depth guarantees (i) that the attraction of the potential increases with the particle size (as one might assume intuitively), (ii) that the interactions are additive in the sense of a Berthelot rule [29], and (iii) that double integrals over $f(\sigma)$ (such as those occurring in the expression for $U^{\mathrm{ex}}$ ) factorize, which makes the calculation of the thermodynamic properties easier. From basic arguments of probability theory, we find that the size distribution $f_{\Sigma}(\sigma)$, along with the $\sigma$ dependence of $\epsilon_{i j}$ [Eq. (42)], induce the following probability distribution for the well depth:

$$
f_{E}(\epsilon)=\frac{1}{z \bar{\epsilon} e^{(-z \bar{\sigma})}} f_{\Sigma}\left[\frac{1}{z} \ln \left(\frac{\epsilon}{\bar{\epsilon}}\right)\right]
$$

The width parameter of the interaction is assumed to be $\sigma$ independent, $\lambda_{i j}=\lambda$; as a consequence, the actual well width $\sigma_{i j} \lambda_{i j}$ increases with the the size of the particles.

To give the reader an idea where these polydisperse systems might be localized in the phase diagram of a corresponding effective one-component system (characterized by the parameters $\eta, \bar{\sigma}, \bar{\eta}$, and $\lambda$ ) we present the approximate values of the critical points of the one-component system, calculated with the same thermodynamic perturbation theory: $\left(\eta_{c} \sim 0.28, \bar{\epsilon}_{c} \sim 1.33\right)$ for system I, and ( $\eta_{c} \sim 0.18, \bar{\epsilon}_{c}$ $\sim 0.8$ ) for system II.

In Fig. 1, we display $f_{\Sigma}\left(\sigma_{i}\right)$ and $\epsilon\left(\sigma_{i}, \sigma_{i}\right)$ as functions of $\sigma_{i}$ for both systems investigated. In an effort to study the convergence of the expansions of the correlation functions in terms of $p_{i}(\sigma)$, we have chosen three different truncation levels $n$ in Eqs. (23) and subsequent expressions, namely, $n$ $=5,8$, and 10. The corresponding zeros of $p_{n}(\sigma), \sigma_{k ; n}$, $k=1, \ldots, n$, are marked in Fig. 1 as vertical lines with different symbols.

\section{Thermodynamic properties}

For a square-well potential, some of the expressions for the thermodynamic properties listed in Sec. II D have to be treated with care. While the internal energy $U^{\mathrm{ex}}$ and the isothermal compressibility $\chi_{T}$ can be calculated via Eqs. (32) and (34) without any problems, the situation for the pressure expression is more delicate; the discontinuity both of the potential and the PDF at contact and at the well edge bring along potential problems. These difficulties were already examined some time ago [30]; following similar lines in the polydisperse case, for the ORPA we obtain the following expression:

$$
\frac{\beta P^{v}}{\rho}=1+\frac{2}{3} \pi \rho \int_{0}^{\infty} d \sigma_{i} d \sigma_{j} f\left(\sigma_{i}\right) f\left(\sigma_{j}\right) \sigma_{i j}^{3}\left\{g\left(\left(\sigma_{i j}\right)^{+} ; \sigma_{i}, \sigma_{j}\right)-\frac{1}{2} \lambda_{i j}^{3} \beta \epsilon_{i j}\left[g\left(\left(\lambda_{i j} \sigma_{i j}\right)^{-} ; \sigma_{i}, \sigma_{j}\right)+g\left(\left(\lambda_{i j} \sigma_{i j}\right)^{+} ; \sigma_{i}, \sigma_{j}\right)\right]\right\},
$$

where the "+" and "-, "superscripts denote the right- and left-side limits, respectively, of the discontinuous function $g\left(r ; \sigma_{i}, \sigma_{j}\right)$ at the specified $r$ values.

\section{Results}

The numerical optimization procedure that finds the minimum of the ORPA functional is based on a simple steepestdescent algorithm (as described, for instance, in Ref. [16]). The search for the minimum starts from the RPA, where the square-well potential is simply extrapolated as a constant into the core region. Minimization of the algorithm is considered to be satisfactory as soon as

$$
\max _{i ; j, k}\left|h\left(r_{i} ; \sigma_{j}, \sigma_{k}\right)\right|<10^{-5} \text { for } r_{i} \epsilon\left[0, \sigma_{i j}\right] .
$$

The optimization procedure turns out to be not too time consuming; even for $n=10$, a satisfactory convergence can be obtained on a Pentium PC $(233 \mathrm{MHz})$ in less than $5 \mathrm{~min}$.

The results for the "diagonal" PDFs $g\left(r ; \sigma_{i}, \sigma_{i}\right)$ and the "diagonal" DCFs $c\left(r ; \sigma_{i}, \sigma_{i}\right)$ are displayed in Figs. 2-5 for systems I and II. In all these figures, we display data for $n$ $=5$. A closer investigation of the $n$ dependence shows that the results for the correlation functions obtained for different $n$ values are consistent, i.e., it is (in principle) possible to interpolate $g\left(r ; \sigma_{i}, \sigma_{i}\right)$ and $c\left(r ; \sigma_{i}, \sigma_{i}\right)$ for the discrete $\sigma_{i}$ by smooth functions $g(r ; \sigma, \sigma)$ and $c(r ; \sigma, \sigma)$. The same holds for the "off-diagonal" correlation functions.

A comparison between the RPA, which violates the core condition, and the ORPA is presented in Figs. 2 (PDF's) and 3 (DCF's); the influence of the optimization criterion that guarantees that the PDF's vanish in the core region is obvious in both functions. On the one hand, the PDF's are now definitely zero inside the core; on the other hand, substantial modifications of the DCF's that represent the optimized potential inside the core are observed. Furthermore, the influence of increasing well depth is clearly visible as $\sigma$ increases; this becomes obvious in the strong modification in $g(r ; \sigma, \sigma)$ as well as in the optimized potential [i.e., the direct correlation functions inside the core, which corrects the unperturbed direct correlation function $\left.c_{0}(r ; \sigma, \sigma)\right]$ as demonstrated in Fig. 5(a).

We also calculate the number-number structure factor $S(q)$

$$
S(q)=1+\rho \int_{0}^{\infty} d \sigma_{i} d \sigma_{j} f\left(\sigma_{i}\right) f\left(\sigma_{j}\right) \tilde{h}\left(q ; \sigma_{i}, \sigma_{j}\right)
$$




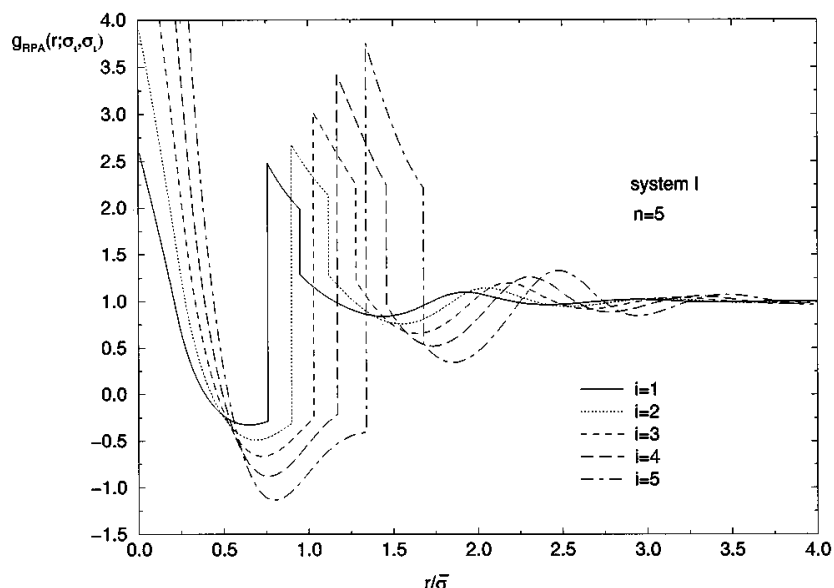

(a)

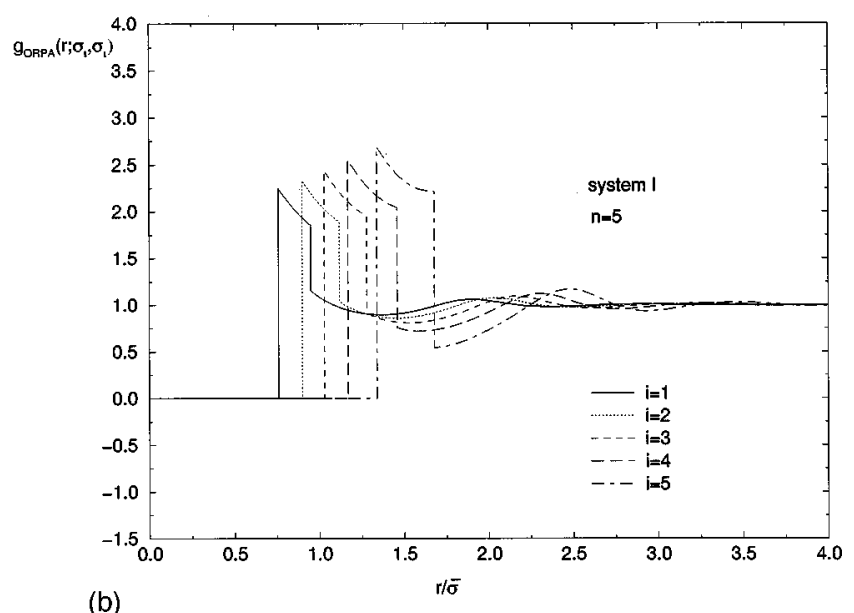

(b)

FIG. 2. "Diagonal" pair distribution functions $g\left(r ; \sigma_{i}, \sigma_{i}\right)$ for the polydisperse system I $(n=5)$ as functions of $r$. Labels for the index $i, i=1, \ldots, 5$, are shown in the inset. (a) RPA results. (b) ORPA results.

This function is displayed, along with the structure factor $S_{0}(q)$ of the reference system, in Figs. 6 and 7 for systems I and II. These figures make visible the influence of the attractive potential. Results obtained from different truncation levels, $n=5,8$, or 10 , coincide within line thickness.

Finally, we compare results for the thermodynamic quantities $U^{\mathrm{ex}}, P^{v}, A^{\mathrm{ex}}$, and $\chi_{T}$ by exploiting the expressions compiled in Secs. II D and III C (also see Table II). Again, we consider three different truncation levels $(n=5,8$, or 10$)$, and also include results for an effective one-component system characterized by the set of system parameters $\eta, \bar{\sigma}, \bar{\epsilon}$, and $\lambda$. For all these quantities, we find that results for different truncation levels differ only by a few percent. Closer investigations (i.e., by solving the $\mathrm{OZ}$ equations for every $n$ value and evaluating the thermodynamic functions) show that a sequence, such as the values $U^{\mathrm{ex}}(n)$, is not monotonous, i.e., the values oscillate. This can be understood by bearing in mind that the zeros $\sigma_{k ; n}$ do not change continuously as one proceeds from one $n$ value to $n-1$. Therefore, in particular for smaller $n$ values, larger differences are observed than is the case for larger $n$ 's. In addition it is obvious that differences in thermodynamic functions become more substantial for different truncation levels $n$ as the distribution $f_{\Sigma}(\sigma)$ becomes broader, i.e., as $\alpha$ decreases (cf. system II).

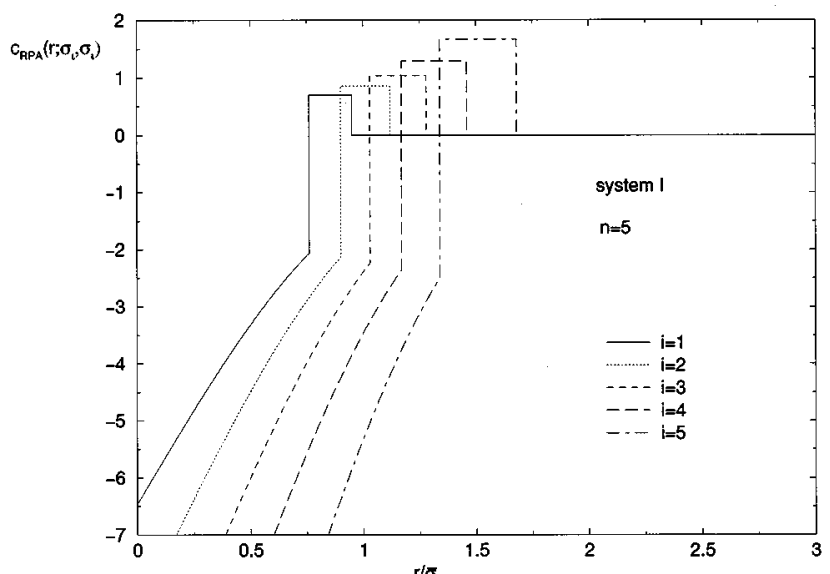

(a)

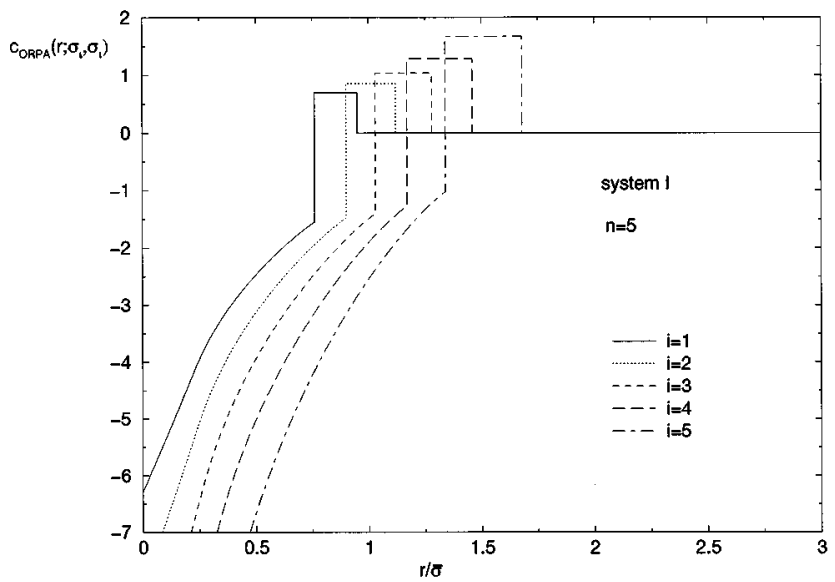

(b)

FIG. 3. "Diagonal" direct correlation functions $c\left(r ; \sigma_{i}, \sigma_{i}\right)$ for the polydisperse system I $(n=5)$ as functions of $r$. Labels for the index $i, i=1, \ldots, 5$, are shown in the inset. (a) RPA results. (b) ORPA results.

Summarizing, and in combination with our observations of the structure factor, we may conclude that a truncation level of $n=5$ or 8 is largely sufficient for reliable numerical results.

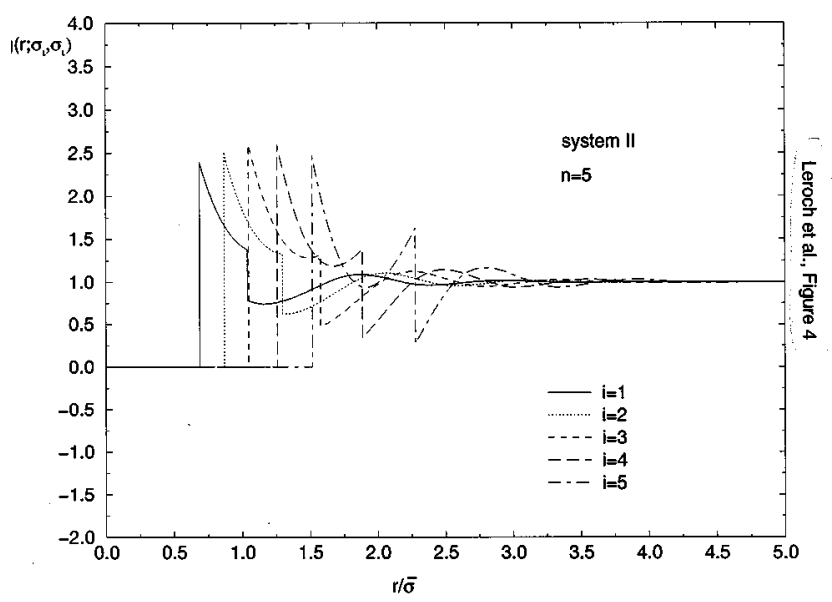

FIG. 4. "Diagonal" pair distribution functions $g\left(r ; \sigma_{i}, \sigma_{i}\right)$ for the polydisperse system II $(n=5)$ as functions of $r$ calculated in the ORPA. Labels for the index $i, i=1, \ldots, 5$, are shown in the inset. 


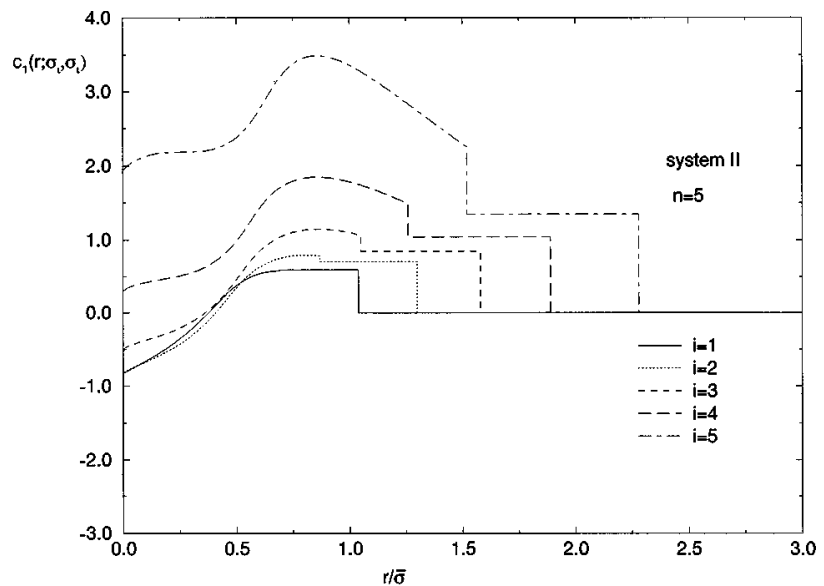

(a)

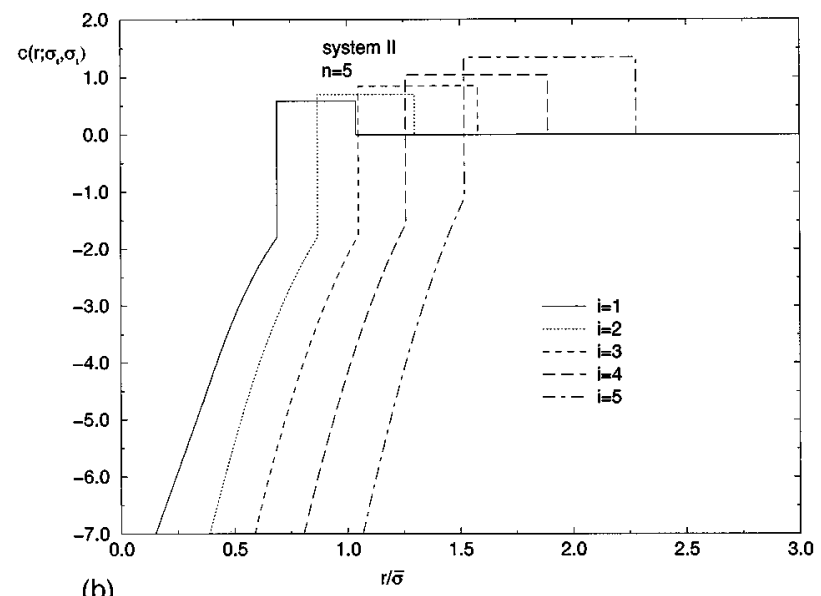

(b)

FIG. 5. (a) Perturbation part of the "diagonal" direct correlation functions $c_{1}\left(r ; \sigma_{i}, \sigma_{i}\right)$ for the polydisperse system II $(n=5)$ as functions of $r$ calculated in the ORPA. Labels for the index $i, i$ $=1, \ldots, 5$, are shown in the inset. (b) "Diagonal'" direct correlation functions $c\left(r ; \sigma_{i}, \sigma_{i}\right)$ for the polydisperse system II $(n=5)$ as functions of $r$ calculated in the ORPA. Labels for the index $i, i$ $=1, \ldots, 5$, are shown in the inset.

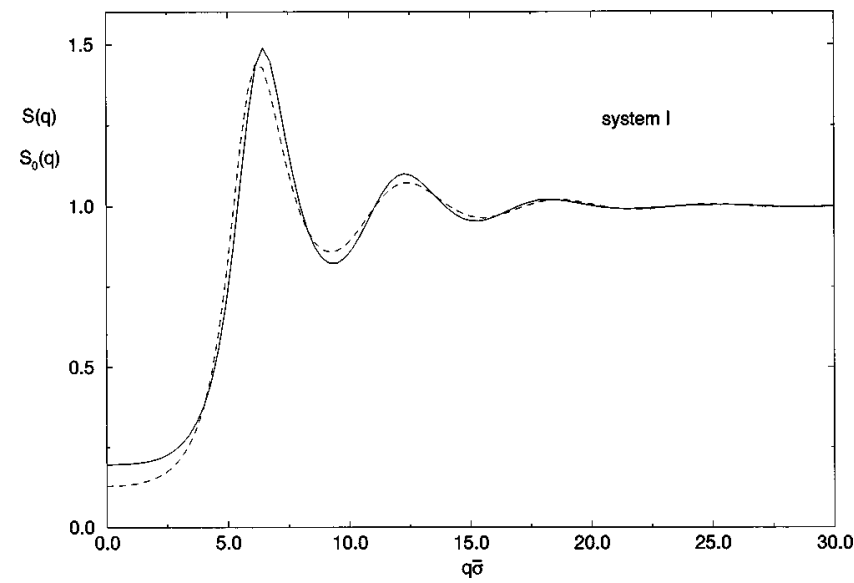

FIG. 6. Number-number structure factors for the polydisperse system I $[S(q)$, full line $]$ and the polydisperse hard sphere reference system $\left[S_{0}(q)\right.$, broken line $]$ as functions of $q$. Results obtained with different truncation levels $n$ coincide within the line thickness.

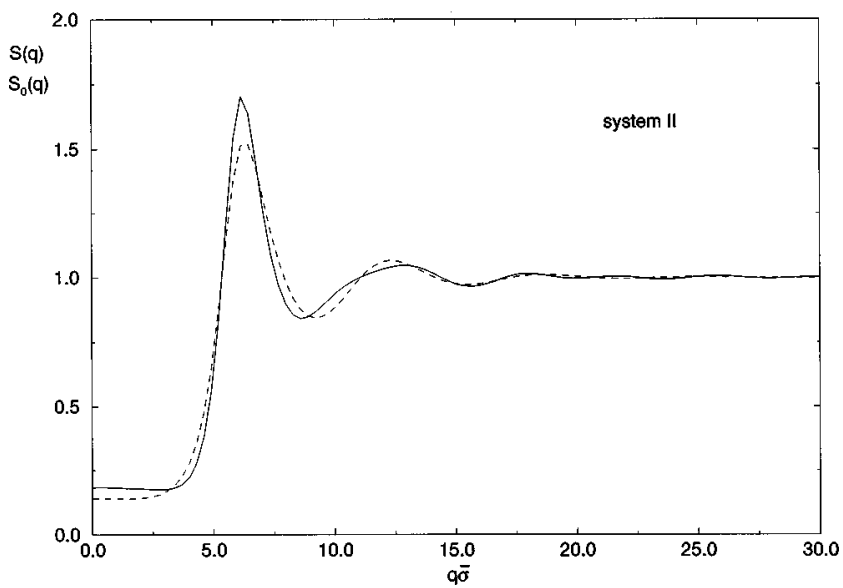

FIG. 7. Number-number structure factors for the polydisperse system II $[S(q)$, full line $]$ and the polydisperse hard sphere reference system $\left[S_{0}(q)\right.$, broken line $]$ as functions of $q$. Results obtained with different truncation levels $n$ coincide within the line thickness.

\section{CONCLUSION}

This work is concerned with a generalization of the optimized random phase approximation (i.e., a thermodynamic perturbation theory) that is able to describe the structure and thermodynamic properties of a polydisperse liquid, where the size distribution of the particles is given by a probability distribution $f_{\Sigma}(\sigma)$. The method is based on expansions of all $\sigma$-dependent functions in terms of the orthonormal polynomials associated with this distribution function. As in the one-component or the (finite) $\mathcal{N}$-component case, the solution of this perturbation theory can be formulated as an optimization problem of a suitably chosen functional of the direct correlation functions; minimization of this functional with respect to variations of the direct correlation functions inside the inaccessible core region defines the solution of the ORPA. To demonstrate the method, we have chosen two polydisperse systems of square-well molecules, assuming a Schulz distribution for the size of the particles and a Berthelot rule for the well depth. A closer analysis of the numerical results for the structure and the thermodynamic properties shows that truncation of the expansion series of the $\sigma$-dependent functions after five terms is in general sufficient.

TABLE II. Thermodynamic properties of systems I and II defined in Table I: excess internal energy $U^{\mathrm{ex}}$, virial pressure $P^{v}$, excess free energy $A^{\text {ex }}$, and isothermal compressibility $\chi_{T}$. (See Secs. II D and III C.) Results are tabulated for series truncation levels $n=5,8$, and 10, as well as for an effective one-component system ("ocs") defined by parameters $\eta, \bar{\sigma}, \bar{\epsilon}$, and $\lambda$.

\begin{tabular}{lccccc}
\hline \hline & & $\beta U^{\mathrm{ex}} / N$ & $\beta P^{v} / \rho$ & $\beta A^{\mathrm{ex}} / N$ & $\chi_{T}$ \\
\hline System I & $n=5$ & -1.6323 & 0.4730 & -0.3228 & 0.3862 \\
& $n=8$ & -1.6442 & 0.4810 & -0.3334 & 0.3878 \\
& $n=10$ & -1.6545 & 0.4860 & -0.3456 & 0.3896 \\
& ocs & -1.6180 & 0.5730 & -0.3709 & 0.3617 \\
\hline \multirow{2}{*}{ System II } & $n=5$ & -2.7694 & 1.0491 & -1.5477 & 0.2066 \\
& $n=8$ & -2.7779 & 1.0657 & -1.5516 & 0.2063 \\
& $n=10$ & -2.7677 & 1.0436 & -1.5326 & 0.2073 \\
& ocs & -2.7344 & 1.2862 & -1.5512 & 0.1776 \\
\hline \hline
\end{tabular}




\section{ACKNOWLEDGMENTS}

This work was supported by the Österreichische Forschungsfond under Project Nos. P11194-PHY and P13062$\mathrm{TPH}$, the Oesterreichische Nationalbank under Project No.
6241, and the Wiener Handelskammer. Computational aid by Andreas Lang is greatfully acknowledged. G.K. is indebted to Dr. Giorgo Pastore (Trieste) for useful hints, helpful discussions, and for providing a copy of his ORPA code.
[1] W. Poon, P. N. Pusey, and H. N. W. Lekkerkerker, Phys. World 9, 27 (1996).

[2] A. Vrij, J. Chem. Phys. 69, 1742 (1978).

[3] L. Blum and G. Stell, J. Chem. Phys. 71, 42 (1979); 72, 2212 (1980).

[4] J. J. Salacuse and G. Stell, J. Chem. Phys. 77, 3714 (1982); J. J. Salacuse, ibid. 81, 2468 (1984); Ph.D. thesis, State University of New York, Stony Brook, 1987 (unpublished).

[5] J. G. Briano and E. D. Glandt, J. Chem. Phys. 80, 3336 (1984).

[6] C. Robertus, W. H. Philipse, J. G. H. Joosten, and Y. K. Levine, J. Chem. Phys. 90, 4482 (1989).

[7] J. N. Herrera and L. Blum, J. Chem. Phys. 94, 5077 (1991); 94, 6190 (1991).

[8] D. Gazzillo, A. Giacometti, and F. Carsughi, J. Chem. Phys. 107, 10141 (1997).

[9] C. Tutschka and G. Kahl, J. Chem. Phys. 108, 9498 (1998).

[10] B. D’Aguanno and R. Klein, Phys. Rev. A 46, 7652 (1992).

[11] F. Lado, Phys. Rev. E 54, 4411 (1996).

[12] F. Lado, Phys. Rev. E 55, 426 (1997); F. Lado, E. Lomba, and M. Lombardero, J. Chem. Phys. 108, 4530 (1998).

[13] F. Lado and E. Lomba, Phys. Rev. Lett. 80, 3535 (1998).

[14] H. C. Andersen and D. Chandler, J. Chem. Phys. 57, 1918 (1972); H. C. Andersen, D. Chandler, and J. D. Weeks, ibid. 56, 3812 (1972).
[15] H. C. Andersen, D. Chandler, and J. D. Weeks, Adv. Chem. Phys. 34, 105 (1976).

[16] G. Pastore, F. Matthews, O. Akinlade, and Z. Badirkhan, Mol. Phys. 84, 653 (1995).

[17] G. Pastore, O. Akinlade, F. Matthews, and Z. Badirkhan, Phys. Rev. E 57, 460 (1998).

[18] A. Lang, C. N. Likos, A. R. Denton, G. Kahl, and H. Löwen (unpublished).

[19] P. Sollich and M. E. Cates, Phys. Rev. Lett. 80, 1365 (1998).

[20] P. B. Warren, Phys. Rev. Lett. 80, 1369 (1998).

[21] J. A. Barker and D. Henderson, J. Chem. Phys. 47, 4714 (1967).

[22] H. C. Andersen, J. D. Weeks, and D. Chandler, Phys. Rev. A 4, 1597 (1971).

[23] J. L. Lebowitz, Phys. Rev. 133, A895 (1964); J. L. Lebowitz and J. S. Rowlinson, J. Chem. Phys. 41, 133 (1964).

[24] L. Verlet and J.-J. Weis, Phys. Rev. A 5, 939 (1972).

[25] E. W. Grundke and D. Henderson, Mol. Phys. 24, 269 (1972).

[26] G. A. Mansoori, N. F. Carnahan, K. E. Starling, and T. W. Leland, J. Chem. Phys. 54, 1523 (1971).

[27] A. Vrij, J. Chem. Phys. 71, 3267 (1979).

[28] G. V. Schulz, Z. Phys. Chem. Abt. B 43, 25 (1939).

[29] J.-P. Hansen and I. R. McDonald, Theory of Simple Liquids, 2nd ed. (Academic, New York, 1986).

[30] W. R. Smith, D. Henderson, and Y. Tago, J. Chem. Phys. 67, 5308 (1977). 